\title{
Photonic reservoir computing approaches to nanoscale computation
}

\author{
Andrew Katumba \\ Ghent University - Photonics \\ Research Group \\ Sint-Pietersnieuwstraat 41 \\ Ghent, Belgium \\ andrew.katumba@ugent.be
}

\author{
Peter Bienstman \\ Ghent University - Photonics \\ Research Group \\ Sint-Pietersnieuwstraat 41 \\ Ghent, Belgium \\ peter.bienstman@ugent.be
}

\author{
Joni Dambre \\ Ghent University - Reservoir \\ Lab \\ Sint-Pietersnieuwstraat 41 \\ Ghent, Belgium \\ joni.dambre@ugent.be
}

\begin{abstract}
This material is based on work in progress. Reservoir computing, originally a training technique for recurrent neural networks, exploits the computation that naturally occurs in physical dynamical systems. Reservoir computing with integrated nanophotonics potentially offers lowpower, high-bandwidth signal processing for telecommunication applications. We present our recent results for optical signal regeneration. Our simulations show that a smallscale low-power integrated photonic reservoir achieves stateof-the-art performance for regenerating optical signals that have traversed fiber lengths of up to $200 \mathrm{~km}$.
\end{abstract}

\section{Categories and Subject Descriptors}

I.5.5 [Computing methodologies]: Pattern RecognitionImplementation, Special Architectures

\section{INTRODUCTION}

At the scaling limits of the traditional MOSFET transistor, the robustness of the digital computing paradigm is faltering. As in the early days of digital computing, alternatives are again being evaluated, but now in view of the recent evolutions of nano-scale integrated technologies. This evolution is one of the drivers behind the renewed interest in analog computing, particularly, biologically inspired computing. Reservoir computing (RC) [4] allows to use the computational power of software recurrent neural networks while avoiding most of the difficulties of training them. In RC, a large nonlinear dynamical system is used for computation. When stimulated with input signals, the system's state has some memory of the input history. Under mild conditions, a broad range of computations can be performed by using optimised linear combinations of the observed system states as outputs. RC can also be applied with a physical dynamical system instead of a software neural network. This has proven a useful framework for analog computing with various physical systems, e.g., mechanical and memristive [2, 3].

Permission to make digital or hard copies of all or part of this work for personal or classroom use is granted without fee provided that copies are not made or distributed for profit or commercial advantage and that copies bear this notice and the full citation on the first page. Copyrights for components of this work owned by others than the author(s) must be honored. Abstracting with credit is permitted. To copy otherwise, or republish, to post on servers or to redistribute to lists, requires prior specific permission and/or a fee. Request permissions from Permissions@acm.org.

NANOCOM' 15 September 21 - 22, 2015, Boston, MA, USA

Copyright is held by the owner/author(s). Publication rights licensed to ACM

ACM 978-1-4503-3674-1/15/09 ... \$15.00

http://dx.doi.org/10.1145/2800795.2800827
Photonics in particular has many potential benefits: very fast nonlinear photonic effects exist, signal propagation is at the speed of light, and photonics lends itself to massive parallelism. Two successful approaches exist. The first uses opto-electronic delayed feedback systems as reservoirs $[1,5]$, emulating a reservoir with many states with a single photonic element. The second approach, followed in this paper, is to construct integrated photonic reservoirs very similar to simulated neural networks [7]. It results in integrated, CMOS-compatible photonic reservoirs that are capable of operating at extremely high bandwidths.

In this paper, we provide our recent progress in nano-photonic reservoir computing. In particular, we present novel simulation results in which it is applied to nonlinear signal regeneration in telecom systems.

\section{NANOPHOTONIC RC}

Integrated photonics is attractive as a platform for photonic reservoir computing as it offers a wide range of optical nonlinearities at different timescales and also presents potential power efficiency benefits over, say, electronic implementations. Using coherent light in reservoir computing takes advantage of both the amplitude and phase. Early work on reservoir computing with integrated photonics [6] demonstrated the promise of this technology. It also ascertained that the restriction to a planar network (the swirl topology, Figure 1(c))) barely affects performance, but that the delays of the interconnections should match the task.

The design with SOA nodes [6] reached state-of-the-art performance on the speech recognition task but consumed a lot of power. Since many tasks do not require very strong nonlinearities, excellent performance can also be achieved with linear passive photonic reservoirs, in which the nonlinearity only comes from the photo-detectors at the readout, which convert complex-valued field values into real-valued intensities [7]. An experimental realisation of a $4 \times 4$ swirl topology passive integrated photonic reservoir was able to perform arbitrary header recognition up to 5 bits [7].

The integrated photonics passive swirl RC design is also used in this work. A crucial advantage is that only one global parameter needs to be optimised at design time: the interconnection delay between the nodes. This parameter also controls the speed at which the reservoir operates.

\section{SIGNAL REGENERATION}

Signal impairments are an inevitability for any kind of communications system, manifested at the receiver as erroneous 
detections that need to be dealt with. In optical fibre systems, these imperfections can mainly be traced back to amplified spontaneous emission at amplification points, attenuation and reflections in fiber links, optical nonlinearities in fibers or timing jitter introduced at $\mathrm{O} / \mathrm{E} \mathrm{E} / \mathrm{O}$ points.

Using nanophotonic reservoir computing, equalization can be performed in the optical domain without incurring an opto-electronic conversion. Moreover, it is conceivable that the reservoir chip could be fabricated on the same design using the same (CMOS compatible) process as the photonics of the receiver yielding significant cost benefits.

Our most recent simulations demonstrate that a $4 \times 4$ passive silicon photonic reservoir suffices for this task. Simulated telecom data was generated with VPI Transmission Maker v9.2 software, using the setup as in figure 1(a). This software incorporates realistic empirical models of the signal degradation in telecom links, caused by the various optical components and the long transmission distance. The data was then used to train and test reservoir designs using inhouse circuit simulation and machine learning libraries. Results are shown in figure 1(b) for a $10 \mathrm{Gbps}$ link. The results indicate BER improvement well below the Forward Error Correction (FEC) limit of $1 \times 10^{-3}$ up to $200 \mathrm{~km}$ of fiber. This means that the chip can be used in conjunction with an appropriately chosen error correction code to achieve error free communication on the link. Such a design would be suitable for signal equalization in, e.g., metro networks.

The results presented in [7] for digital tasks and in this pa-

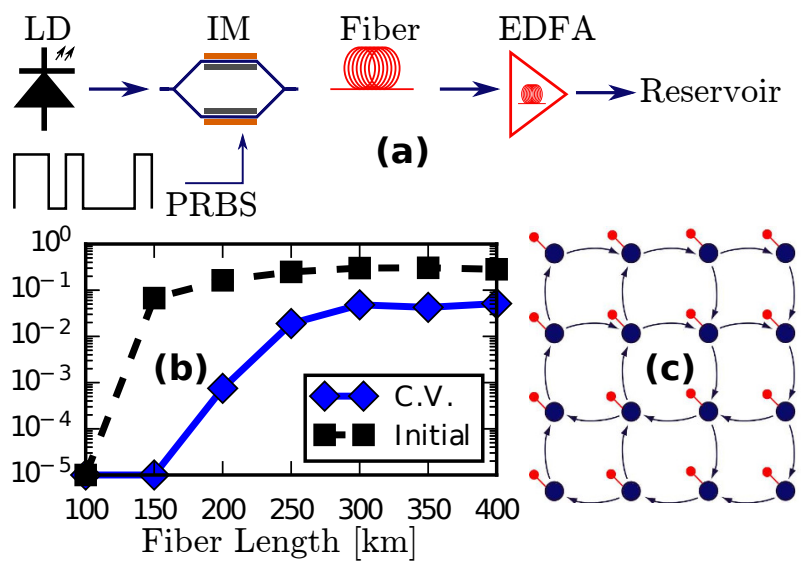

Figure 1: (a) Schematic of simulation setup for signal equalization task (b) Initial BER rates and average cross validation (C.V.) BER rates for fiber links up to $400 \mathrm{~km}$ (c) The $4 \times 4$ swirl reservoir topology.

per for an analog task demonstrate the promise of nanophotonic reservoir computing. It opens up new opportunities for performing some of the high-bandwidth operations, typically found in telecom applications, directly on optical signals. In order to bring this technology to the market, we are currently addressing some the remaining challenges. A first challenge is the scalability of passive photonic reservoirs. While they are more power efficient than reservoirs with active nodes, due to losses in the system, the optical signals do not travel far enough to use the full computational richness of larger reservoirs. We are therefore addressing technological solutions to reduce the losses in the system and studying alternative architectures in which optical gain is introduced very sparsely. A second challenge is the integrated implementation of the readout. An integrated readout that directly implements our current approach would require an expensive integrated high-bandwidth photodetector for each observed state, which is both power and cost inefficient. We are currently reducing the number of required photodetectors by implementing a readout in the optical domain. This implies that the training algorithm will no longer have direct access to the reservoir states. We are therefore also exploring new training approaches that can deal with this.

\section{CONCLUSION}

This paper extends our work on physical reservoir computing with nanophotonics to high-bandwidth nonlinear signal regeneration. Simulation results show that a small passive integrated photonic reservoir can reduce the BER with several orders of magnitude for fiber lengths up to $200 \mathrm{~km}$ and almost one order of magnitude for lengths up to $400 \mathrm{~km}$. Two major challenges remain for industrial uptake of the technology: scalability to larger reservoirs, as is required for more complex tasks, and hardware-friendly readout and training. Given the fact that photonics becomes very powerefficient at high bandwidths and that integrated nanophotonics in silicon are compatible with traditional CMOS processing, we believe that this technology will evolve into a viable candidate for next-generation telecom systems.

\section{ACKNOWLEDGEMENTS}

This research was funded by the ERC starting grant Naresco, the BELSPO IAP P7-35 program photonics@be and by the the EU FP7 Human Brain Project (Grant No 604102).

\section{REFERENCES}

[1] D. Brunner, M. C. Soriano, C. R. Mirasso, and I. Fischer. Parallel photonic information processing at gigabyte per second data rates using transient states. Nature Communications, 4, 2012.

[2] H. Hauser, A. J. Ijspeert, R. M. Füchslin, R. Pfeifer, and W. Maass. Towards a theoretical foundation for morphological computation with compliant bodies. Biological Cybernetics, 105:355-370, Jan. 2012.

[3] M. Kulkarni and C. Teuscher. Memristor-based reservoir computing. In Nanoscale Architectures (NANOARCH), 2012 IEEE/ACM International Symposium on, pages 226-232, July 2012.

[4] M. Lukoševičius and H. Jaeger. Reservoir computing approaches to recurrent neural network training. Computer Science Review, 3:127-149, 2009.

[5] Y. Paquot, F. Duport, A. Smerieri, J. Dambre, B. Schrauwen, M. Haelterman, and S. Massar. Optoelectronic reservoir computing. Scientific Reports, $2: 287: 1-6,2012$.

[6] K. Vandoorne, J. Dambre, D. Verstraeten, B. Schrauwen, and P. Bienstman. Parallel reservoir computing using optical amplifiers. IEEE Transactions on Neural Networks, 22(9):1469-1481, 2011.

[7] K. Vandoorne, P. Mechet, T. Van Vaerenbergh, M. Fiers, G. Morthier, D. Verstraeten, B. Schrauwen, J. Dambre, and P. Bienstman. Experimental demonstration of reservoir computing on a silicon photonics chip. Nature communications, 5:3541, Jan. 2014. 